\title{
An effective approximation for singularly perturbed problem with multi-point boundary value
}

Derya Arslan

Department of Mathematics, Faculty of Art and Science, Bitlis Eren University, Bitlis, Turkey

Received: 12 January 2021, Accepted: 11 April 2021

Published online: 18 April 2021.

\begin{abstract}
This study deals with the singularly perturbed multi-point boundary value problem and an effective numerical method. The method analysis singularly perturbed problem with multi-point boundary value as theoretically and experimentally. It is shown that the presented method has first-order approximation in the discrete maximum norm. The numerical results are presented in table and graphs, and these results come out the validity of the theoretical analysis of our method.
\end{abstract}

Keywords: Singular perturbation, Finite difference method, Shishkin mesh, Uniformly convergence, Multi-point condition.

\section{Introduction}

Consider the following second-order linear singularly perturbed multi-point boundary value problem

$$
\begin{gathered}
-\varepsilon u^{\prime \prime}+b(x) u(x)=f(x), \quad 0<x<1, \\
u(0)=0 \\
u(1)=\sum_{i=1}^{m} c_{i} u\left(s_{i}\right)+d,
\end{gathered}
$$

where $0<\varepsilon \ll 1$ is a small perturbation parameter; $b, d, m$ and $c_{i}$ are given constants, $0<s_{i}<1, i=1,2, \ldots m$; and $b(x) \geq b^{2}>0$ and $f(x)$ are assumed to be continuous functions in $[0,1]$, and moreover

$$
\begin{gathered}
-\infty<\sum_{i=1}^{m} c_{i} w_{0}\left(s_{i}\right)<1, \\
w_{0}(x)=\frac{1-e^{-\frac{2 b x}{\sqrt{\varepsilon}}}}{1-e^{-\frac{2 b}{\sqrt{\varepsilon}}}} e^{\frac{b(x-1)}{\sqrt{\varepsilon}}} .
\end{gathered}
$$

It is a well known fact that differential equations with a small parameter $\varepsilon$ multiplying the highest-order derivative terms are called singularly perturbed differential equations. Standard numerical methods for solving singularly perturbed problems are fail to give accurate results and unstable due to the perturbation parameter $\varepsilon$. Therefore, there are some fitted numerical methods to solve equations like these, such finite difference methods, finite element methods etc. So, we prefer to use finite difference method for solving this problem in this paper. 
Singular perturbation problems arise in chemical-reactor theory, control theory, oceanography, fluid mechanics, quantum mechanics, hydro mechanical problems, meteorology, electrical networks and other physical models $[13,14,17,18,19$, $20,21,22]$. Singularly perturbed differential equations with nonlocal boundary value have been studied by many authors. According to some references, existence and uniqueness of nonlocal problems can be seen in [1,4,22]. A finite difference scheme on an uniform mesh for solving linear (nonlinear) singularly perturbed problem with nonlocal condition have been found in $[1,2,3,5,6,7,8,9,10,11,12,15,16]$.

In the above aforementioned papers, related studies to singularly perturbed problems are related only with the ordinary cases. In addition, available studies for the numerical solution of singularly perturbed problems with multi-point boundary conditions have not widespread yet. It can be seen in $[5,10]$ that various difference schemes exist for multi-point and integral boundary conditions.

In this present paper, we use finite difference method on a Shishkin mesh for the numerical solution of the nonlocal problem (1)-(3). This method is shown uniformly convergent of first-order on Shishkin mesh, in discrete maximum norm. Some properties of the exact solution of the problem described in (1)-(3) is investigated in Section 2. Finite difference schemes on Shishkin mesh for the problem (1)-(3) are constructed in Section 3. The error analysis for the difference scheme is performed in Section 4. Finally, We formulate the iterative algorithm for solving the discrete problem and a numerical example present to find the solution of approximation in Section 5.

Henceforth, $C, C_{0}$ and $C_{1}$ will mean positive constants independent of $\varepsilon$ and the mesh parameter.

\section{Some properties of the continuous problem}

Here we establish very important asymptotic properties of the exact solution of the problem (1)-(3) that will be used to anaylze appropriate finite difference problem.

Lemma 1. If $b(x)$ and $f(x)$ be sufficiently smooth on interval $[0,1]$ and

$$
\sum_{i=1}^{m} c_{i} w\left(s_{i}\right)<1
$$

where $w_{0}(x) \geq|w(x)|$ is the solution of the following problem

$$
\begin{gathered}
-\varepsilon w^{\prime \prime}+b(x) w(x)=0, \\
w(0)=0, w(1)=1 .
\end{gathered}
$$

Then, the solution of the problem (1)-(3) satisfies the following inequalities:

$$
\|u(x)\|_{C[0,1]} \leq C_{0}
$$

where

$$
C_{0}=|v(x)|+|\lambda||w(x)|
$$

and

$$
\left|u^{\prime}(x)\right| \leq C_{1}\left\{1+\frac{1}{\sqrt{\varepsilon}}\left(e^{-\frac{b x}{\sqrt{\varepsilon}}}+e^{-\frac{b(1-x)}{\sqrt{\varepsilon}}}\right)\right\}, \quad 0<x<1
$$


Proof. Let us take $u(1)=\lambda$ and the solution of the problem (1)-(3) as

$$
u(x)=v(x)+\lambda w(x)
$$

where

$$
\lambda=\frac{d+\sum_{i=1}^{m} c_{i} v\left(s_{i}\right)}{1-\sum_{i=1}^{m} c_{i} w\left(s_{i}\right)},
$$

and the function $v(x)$ is the solution of the following problem

$$
\begin{gathered}
-\varepsilon v^{\prime \prime}+b(x) v(x)=f(x), \\
v(0)=0, v(1)=0 .
\end{gathered}
$$

Now, we use the maximum principle for the evaluation of the functions $v(x)$ and $w(x)$, and so we have

$$
|v(x)| \leq|v(0)|+|v(1)|+b^{-2}\|f\|_{C[0,1]} \leq b^{-2}\|f\|_{C[0,1]} \leq C_{1}
$$

and

$$
|w(x)| \leq|w(0)|+|w(1)| \leq 1
$$

Finally, from (7) and (8), we obtain

$$
|u(x)|=|v(x)|+|\lambda||w(x)| \leq C_{1}+|\lambda| \leq C_{0},
$$

which proves (5).

Next, we will examine the inequality (6). Differentiating the Equation (1), we get the relation

$$
-\varepsilon u^{\prime \prime \prime}(x)+b(x) u^{\prime}(x)=\Phi(x)
$$

where

$$
\Phi(x)=f^{\prime}(x)-b^{\prime}(x) u(x)
$$

After doing some calculation in the Equation (9), we obtain

$$
\left|u^{\prime}(x)\right| \leq C+\frac{C}{\sqrt{\varepsilon}}\left(e^{-\frac{b x}{\sqrt{\varepsilon}}}+e^{-\frac{b(1-x)}{\sqrt{\varepsilon}}}\right) \leq C\left\{1+\frac{1}{\sqrt{\varepsilon}}\left(e^{-\frac{b x}{\sqrt{\varepsilon}}}+e^{-\frac{b(1-x)}{\sqrt{\varepsilon}}}\right)\right\}
$$

(see in [8]). Eventually, we have the inequality (6). And so, the proof of Lemma 1 is completed.

\section{The Establishment of difference scheme}

In here, we discretize the problem (1)-(3) using a finite difference method on a piecewise uniform mesh of Shishkin type. The Shishkin mesh is introduced for this study as follows. 


\subsection{Shishkin mesh}

The approximation to the solution $u$ of the problem (1)-(3) will be computed on a Shishkin mesh. For a divisible by four positive integer $N$, we divide the interval $[0,1]$ into the three subintervals $[0, \sigma],[\sigma, 1-\sigma]$ and $[1-\sigma, 1]$. In practice, we usually has $\sigma \ll 1$, and so the mesh is fine on the intervals $[0, \sigma]$ and $[1-\sigma, 1]$ and coarse on the interval $[\sigma, 1-\sigma]$. Here $\sigma$ is transition point which is called as following:

$$
\sigma=\min \left\{\frac{1}{4}, b^{-1} \varepsilon \ln N\right\}
$$

We introduce a set of the mesh points $\bar{\omega}_{N}=\left\{x_{i}\right\}_{i=0}^{N}$,

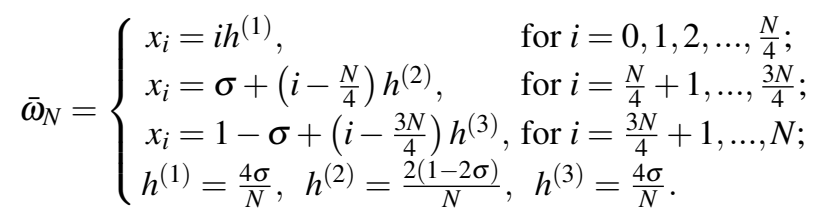

$$
\begin{aligned}
& h^{(2)}+\frac{1}{2}\left(h^{(1)}+h^{(3)}\right)=\frac{2}{N}, h^{(k)} \leq N^{-1}, k=1,3, N^{-1} \leq h^{(2)} \leq 2 N^{-1} .
\end{aligned}
$$

For each $i \geq 1$ we set the step-size $h_{i}=x_{i}-x_{i-1}, i=1,2, \ldots, N$.

\subsection{Construction of the difference scheme on Shishkin mesh}

We introduce an any non-uniform mesh on the interval $[0,1]$

$$
\omega_{N}=\left\{0<x_{1}<x_{2}<\ldots<x_{N-1}<1\right\},
$$

and

$$
\bar{\omega}_{N}=\omega_{N} \cup\left\{x_{0}=0, x_{N}=1\right\} .
$$

Before describing our numerical method, we introduce some notations for the mesh functions. We define the following finite difference for any mesh function $g_{i}=g\left(x_{i}\right)$ given on $\bar{\omega}_{N}$ :

$$
\begin{gathered}
g_{\bar{x}, i}=\frac{g_{i}-g_{i-1}}{h_{i}}, g_{x, i}=\frac{g_{i+1}-g_{i}}{h_{i+1}}, g_{x, i}=\frac{g_{x, i}+g_{\bar{x}, i}}{2}, \\
g_{\widehat{x}, i}=\frac{g_{i+1}-g_{i}}{\hbar_{i}}, g_{\widehat{x}, i}=\frac{g_{x, i}-g_{\bar{x}, i}}{\hbar_{i}}, \hbar_{i}=\frac{h_{i}+h_{i+1}}{2}, \\
\|g\|_{\infty} \equiv\|g\|_{\infty, \bar{\omega}_{N}}:=\max _{0 \leqslant i \leqslant N}\left|g_{i}\right| .
\end{gathered}
$$

Now, we construct the difference scheme for the Equation (1). Firstly, we will integrate the Equation (1) over $\left(x_{i-1}, x_{i+1}\right)$,

$$
\delta_{i} \hbar_{i}^{-1} \int_{x_{i-1}}^{x_{i+1}} L u(x) \varphi_{i}(x) d x=\delta_{i} \hbar_{i}^{-1} \int_{x_{i-1}}^{x_{i+1}} f(x) \varphi_{i}(x) d x, i=1, \ldots N
$$


here $\left\{\varphi_{i}(x)\right\}_{i=1}^{N-1}$ is the basis functions that $\left\{\varphi_{i}(x)\right\}_{i=1}^{N-1}$ has the following form

$$
\varphi_{i}(x)= \begin{cases}\varphi_{i}^{(1)}(x)=\frac{\operatorname{sh} \gamma_{i}\left(x-x_{i}\right)}{s h \gamma_{i} h}, & x_{i-1}<x<x_{i}, \\ \varphi_{i}^{(2)}(x)=\frac{\operatorname{sh} \gamma_{i}\left(x_{i+1}-x\right)}{\operatorname{sh} \gamma_{i} h}, & x_{i}<x<x_{i+1}, \\ 0, & x \notin\left(x_{i-1}, x_{i+1}\right),\end{cases}
$$

where $\gamma_{i}=\sqrt{\frac{b_{i}}{\varepsilon}} ; \varphi_{i}^{(1)}(x)$ and $\varphi_{i}^{(2)}(x)$, respectively, are the solution of the problems as:

$$
\begin{gathered}
-\varepsilon \varphi^{\prime \prime}+b_{i} \varphi=0, \quad x_{i-1}<x<x_{i}, \\
\varphi\left(x_{i-1}\right)=0, \quad \varphi\left(x_{i}\right)=1 . \\
-\varepsilon \varphi^{\prime \prime}+b_{i} \varphi=0, \quad x_{i}<x<x_{i+1}, \\
\varphi\left(x_{i}\right)=1, \quad \varphi\left(x_{i+1}\right)=0 .
\end{gathered}
$$

After doing some arrangements in the Equation (11), we have

$$
\delta_{i} \hbar_{i}^{-1} \int_{x_{i-1}}^{x_{i+1}} u^{\prime}(x) \varphi^{\prime}{ }_{i}(x) d x+\delta_{i} b_{i} \hbar_{i}^{-1} \int_{x_{i-1}}^{x_{i+1}} u(x) \varphi_{i}(x) d x=f_{i}+R_{i}
$$

where

$$
R_{i}=\delta_{i} \hbar_{i}^{-1} \int_{x_{i-1}}^{x_{i+1}}\left[b\left(x_{i}\right)-b(x)\right] u(x) \varphi_{i}(x) d x+\delta_{i} \hbar_{i}^{-1} \int_{x_{i-1}}^{x_{i+1}}\left[f(x)-f\left(x_{i}\right)\right] \varphi_{i}(x) d x
$$

and

$$
\delta_{i}=\left(\hbar_{i}^{-1} \int_{x_{i-1}}^{x_{i+1}} \varphi_{i}(x) d x\right)^{-1}
$$

Using the interpolating quadrature rules (2.1) and (2.2) from [4] with weight functions $\varphi_{i}(x)$ on subintervals $\left(x_{i-1}, x_{i+1}\right)$ from (12), we obtain the following precise relation:

$$
l u_{i}:=-\varepsilon \theta_{i} u_{\widehat{x} \widehat{x}, i}+b_{i} u_{i}=f_{i}+R_{i}, i=1, \ldots, N
$$

where

$$
\theta_{i}=\frac{b_{i} h_{i}^{2}}{4 \varepsilon s h^{2}\left(\frac{\gamma_{i} h_{i}}{2}\right)} .
$$

If we neglect $R_{i}$ in the Equation (14), we can suggest the following difference scheme for the problem (1)-(3):

$$
\begin{gathered}
l y_{i}:=-\varepsilon \theta_{i} y_{\widehat{x} \widehat{x}, i}+b_{i} y_{i}=f_{i}, i=1, \ldots, N, \\
y_{0}=0, \\
y_{N}=\sum_{i=1}^{m} c_{i} y_{N_{i}}\left(x_{N_{i}}\right)+d,
\end{gathered}
$$

where $x_{N_{i}}$ is the mesh point nearest to $s_{i}$, and $\theta_{i}$ is given by (15). 


\section{Uniform error estimates}

In this part, we will investigate the convergence of the method for the problem (1) and (3). We will give the error function $z_{i}=y_{i}-u_{i}, i=0,1, \ldots, N$, where $z_{i}$ is the solution of the discrete problem

$$
\begin{gathered}
-\varepsilon \theta_{i} z_{\widehat{x} \widehat{x}, i}+b_{i} z_{i}=R_{i}, i=1, \ldots, N, \\
z_{0}=0, \\
z_{N}=\sum_{i=1}^{m} c_{i} z_{N_{i}},
\end{gathered}
$$

where $R_{i}$ and $\theta_{i}$ are defined by (13) and (15), respectively.

Lemma 2. Let $z_{i}$ be the solution (19)-(21) and

$$
\sum_{i=1}^{m} c_{i} z_{2}\left(s_{i}\right) \neq 1
$$

Then the estimate

$$
\|z\|_{\infty, \bar{\omega}_{N}} \leq C\|R\|_{\infty, \omega_{N}}
$$

holds.

Proof. Let $z(x)=z_{1}(x)+\lambda z_{2}(x)$ be the solution of the discrete problem (19)-(21), where $z_{1}(x)$ and $z_{2}(x)$ are the solution of the following problems, respectively:

$$
\begin{gathered}
-\varepsilon \theta_{i} z_{\bar{x} \widehat{x}, i}+b_{i} z_{i}=R_{i}, i=1, \ldots, N, \\
z_{1}(0)=0, z_{1}(1)=0,
\end{gathered}
$$

and

$$
\begin{gathered}
-\varepsilon \theta_{i} z_{\widehat{x} \widehat{x}, i}+b_{i} z_{i}=0, i=1, \ldots, N, \\
z_{2}(0)=0, z_{2}(1)=1,
\end{gathered}
$$

where

$$
\begin{array}{r}
\lambda=\frac{d+\sum_{i=1}^{m} c_{i} z_{1}\left(s_{i}\right)}{1-\sum_{i=1}^{m} c_{i} z_{2}\left(s_{i}\right)}, \\
1-\sum_{i=1}^{m} c_{i} z_{2}\left(s_{i}\right) \neq 0 .
\end{array}
$$

According to the maximum principle for $z_{1}(x)$ and $z_{1}(x)$, we have the following evaluations:

$$
\left|z_{1}(x)\right| \leq\left|z_{1}(0)\right|+\left|z_{1}(1)\right|+b^{-2}\|R\|_{\infty, \omega_{N}} \leq C\|R\|_{\infty, \omega_{N}},
$$

and

$$
\left|z_{2}(x)\right| \leq\left|z_{2}(0)\right|+\left|z_{2}(1)\right| \leq 1
$$


Next, we have from (23) and (24)

$$
|z(x)| \leq\left|z_{1}(x)\right|+|\lambda|\left|z_{2}(x)\right| \leq b^{-2}\|R\|_{\infty, \omega_{N}}+|\lambda| \leq C\|R\|_{\infty, \omega_{N}},
$$

which proves Lemma 2.

Lemma 3. Under the assumptions of section 1 and Lemma 2 the solution of the problem (1)-(3) satisfies the following estimates for the remainder term $R_{i}$ :

$$
\|R\|_{\infty, \bar{\omega}_{N}} \leq C N^{-1} \ln N
$$

Proof. The reminder term $R_{i}$ can be rewritten with (2) and using mean value theorem as

$$
\left|R_{i}\right| \leq \delta_{i} \hbar_{i}^{-1} \int_{x_{i-1}}^{x_{i+1}} C h_{i} C_{0} \varphi_{i}(x) d x+\delta_{i} \hbar_{i}^{-1} \int_{x_{i-1}}^{x_{i+1}} C h_{i} \varphi_{i}(x) d x \leq C h_{i}
$$

Now, we will evaluate $R_{i}$ for the intervals $[0, \sigma],[\sigma, 1-\sigma]$ and $[1-\sigma, 1]$, respectively. In the first case, for $\frac{1}{4}>b^{-1} \varepsilon \ln n=\sigma$ and the interval $[0, \sigma]$ :

$$
\left|R_{i}\right| \leq C h^{(1)} \leq \frac{4 C \sigma}{N} \leq \frac{4 b^{-1} \varepsilon \ln N}{N} \leq C N^{-1} \ln N, \quad i=1, \ldots, \frac{N}{4}-1 .
$$

In the second case, for $\frac{1}{4}>b^{-1} \varepsilon \ln n=\sigma$ and the interval $[\sigma, 1-\sigma]$ :

$$
\left|R_{i}\right| \leq C h^{(2)}=\frac{2 C(1-2 \sigma)}{N}=\frac{2 C\left(1-2 b^{-1} \varepsilon \ln N\right)}{N} \leq C N^{-1} \ln N, \quad i=\frac{N}{4}+1, \ldots, \frac{3 N}{4}-1 .
$$

In the third case, for $\frac{1}{4}>b^{-1} \varepsilon \ln n=\sigma$ and the interval $[1-\sigma, 1]$ :

$$
\left|R_{i}\right| \leq C h^{(3)}=\frac{4 C \sigma}{N} \leq \frac{4 b^{-1} \varepsilon \ln N}{N} \leq C N^{-1} \ln N, \quad i=\frac{3 N}{4}+1, \ldots, N
$$

and then, we evaluate $R_{i}$ for $i=\frac{N}{4}$ and $i=\frac{3 N}{4}$, respectively:

$$
\left|R_{\frac{N}{4}}\right| \leq C h=C h^{(1)}=C 4 \sigma N^{-1} \leq 4 C N^{-1} b^{-1} \varepsilon \ln N \leq C N^{-1} \ln N,
$$

and

$$
\left|R_{\frac{3 N}{4}}\right| \leq C h=C h^{(2)}=2 C\left(1-2 b^{-1} \varepsilon \ln N\right) N^{-1} \leq C N^{-1} \ln N .
$$

According to all these situations, we have

$$
\left|R_{i}\right| \leq C h_{i} \leq C N^{-1} \ln N
$$

This completes the proof of Lemma 3.

We can state the convergence result of this study the following Theorem 4.

Theorem 1. Let $u(x)$ be the solution of the problem (1)-(3) and $y_{i}$ be the solution of the difference scheme (16)-(18). Then, the following uniform error estimate satisfies

$$
\|y-u\|_{\infty, \bar{\omega}_{N}} \leq C N^{-1} \ln N .
$$




\section{Algorithm and numerical results}

Here an effective algorithm has been given for the solution of the difference scheme (16)-(18) and numerical results have also been displayed in table and graphs.

(a) We give the algorithm for the solution of the difference scheme (16)-(18):

$$
\begin{gathered}
\left(\frac{\varepsilon \theta_{i}}{\hbar_{i} h_{i}}\right) y_{i-1}-\left(\frac{2 \varepsilon \theta_{i}}{h_{i} h_{i+1}}+b_{i}\right) y_{i}+\left(\frac{\varepsilon \theta_{i}}{\hbar_{i} h_{i+1}}\right) y_{i+1}=-f_{i}, \quad i=1, \ldots, N-1, \\
A_{i}=\frac{\varepsilon \theta_{i}}{\hbar_{i} h_{i}}, B_{i}=\frac{\varepsilon \theta_{i}}{\hbar_{i} h_{i+1}}, C_{i}=\frac{2 \varepsilon \theta_{i}}{h_{i} h_{i+1}}+b_{i}, \\
\alpha_{1}=0, \beta_{1}=0, \alpha_{\frac{N}{10}+1}=0, \beta_{\frac{N}{10}+1}=\mu_{1}, \\
\alpha_{\frac{2 N}{10}+1}=0, \beta_{\frac{2 N}{10}+1}=\mu_{2}, \alpha_{\frac{5 N}{10}+1}=0, \beta_{\frac{5 N}{10}+1}=\mu_{3}, \\
\alpha_{\frac{9 N}{10}+1}=0, \beta_{\frac{9 N}{10}+1}=\mu_{4}, \alpha_{i+1}=\frac{B_{i}}{C_{i}-A_{i} \alpha_{i}}, \beta_{i+1}=\frac{F_{i}+A_{i} \beta_{i}}{C_{i}-A_{i} \alpha_{i}}, i=1, \ldots, N-1, \\
y_{i}^{(n)}=\alpha_{i+1} y_{i+1}^{(n)}+\beta_{i+1}, i=N-1, \ldots, 2,1 .
\end{gathered}
$$

(b) We examine the following problem to see how the method works:

$$
\begin{gathered}
-\varepsilon u^{\prime \prime}(x)+u(x)=-\cos ^{2}(\pi x)-2 \varepsilon \pi^{2} \cos (2 \pi x), 0<x<1, \\
u(0)=0, \quad u(1)=0.03 u(0.9)+0.2 u(0.1)+0.5 u(0.2)+0.09 u(0.5)+d,
\end{gathered}
$$

where

$$
d=0.192+\frac{0.4415 e^{\frac{1}{\sqrt{\varepsilon}}}}{1+e^{-\frac{1}{\sqrt{\varepsilon}}}}
$$

We have the exact solution of this problem as

$$
u(x)=\frac{\exp \left(-\frac{x}{\sqrt{\varepsilon}}\right)+\exp \left(\frac{x-1}{\sqrt{\varepsilon}}\right)}{1+\exp \left(-\frac{1}{\sqrt{\varepsilon}}\right)}-\cos ^{2}(\pi x) .
$$

The corresponding $\varepsilon$ - uniform convergence rates are computed using the formula

$$
P^{N}=\frac{\ln \left(e^{N} / e^{2 N}\right)}{\ln 2} .
$$

The error estimates are denoted by

$$
e^{N}=\max _{\varepsilon} e_{\varepsilon}^{N}, \quad e_{\varepsilon}^{N}=\|y-u\|_{\infty, \bar{\omega}_{N}} .
$$

\section{Conclusion}

In this study, we have offered an effective finite difference method for solving second-order linear singularly perturbed multi-point boundary value problem. The method has display uniform convergence with respect to the perturbation 
Table 1: The computed maximum pointwise errors $e^{N}$ and rates of convergence $p^{N}$

\begin{tabular}{clllllll}
\hline$\varepsilon$ & $N=20$ & $N=40$ & $N=80$ & $N=160$ & $N=320$ & $N=640$ & $N=1280$ \\
\hline $2^{-4}$ & 0.021418 & 0.006108 & 0.002110 & 0.0010691 & 0.000537 & 0.000269 & 0.000135 \\
& 1.81 & 1.53 & 0.98 & 0.99 & 0.99 & 0.99 & 1.01 \\
$2^{-5}$ & 0.043843 & 0.021665 & 0.011007 & 0.005546 & 0.002741 & 0.001317 & 0.000614 \\
& 1.01 & 0.97 & 0.98 & 1.01 & 1.05 & 1.09 & 1.15 \\
$2^{-6}$ & 0.058022 & 0.027151 & 0.014254 & 0.007787 & 0.004269 & 0.002315 & 0.001237 \\
& 1.09 & 0.92 & 0.87 & 0.86 & 0.88 & 0.90 & 0.92 \\
$2^{-7}$ & 0.071795 & 0.029787 & 0.014522 & 0.008021 & 0.004490 & 0.002520 & 0.001403 \\
& 1.26 & 1.03 & 0.85 & 0.83 & 0.83 & 0.84 & 0.83 \\
$2^{-8}$ & 0.096957 & 0.033896 & 0.014905 & 0.007592 & 0.004166 & 0.002377 & 0.001323 \\
& 1.51 & 1.18 & 0.97 & 0.86 & 0.81 & 0.84 & 0.83 \\
\hline$e^{N}$ & 0.096957 & 0.033896 & 0.014905 & 0.008021 & 0.004490 & 0.002520 & 0.001403 \\
$p^{N}$ & 1.51 & 1.18 & 0.97 & 0.83 & 0.83 & 0.84 & 0.83 \\
\hline
\end{tabular}

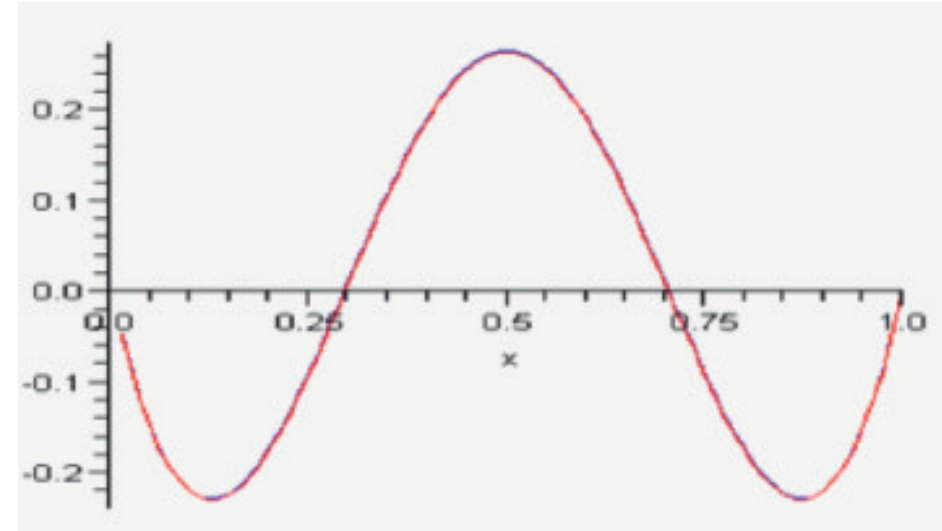

Fig. 1: Comparison of approximate solution and exact solution for $\mathrm{N}=80, \varepsilon=2^{-4}$.

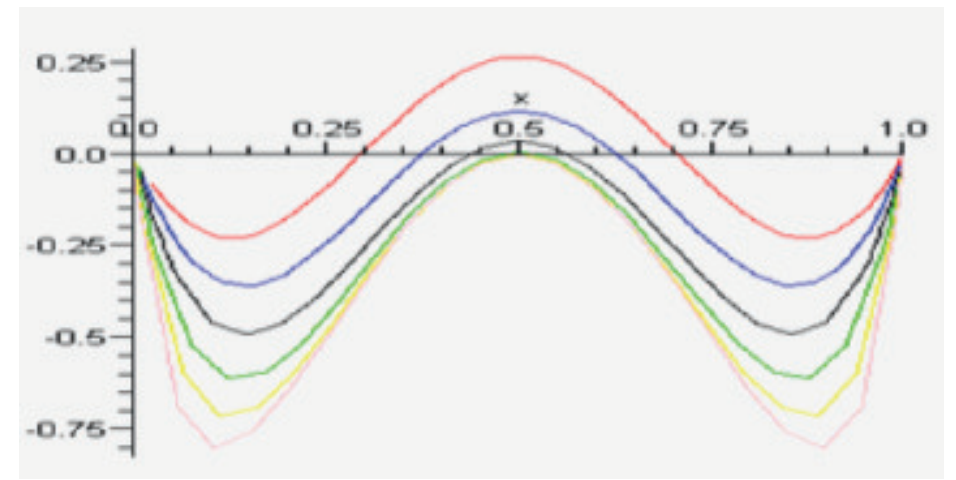

Fig. 2: Exact solution distribution for $\mathrm{N}=40, \varepsilon=2^{-4}, \ldots, 2^{-8}$. 


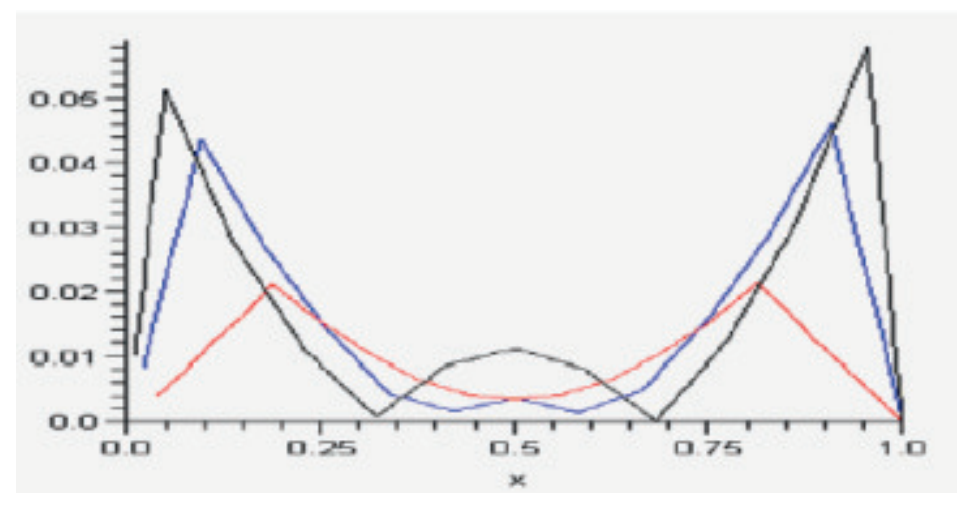

Fig. 3: Error distribution for $\mathrm{N}=20, \varepsilon=2^{-4}, \varepsilon=2^{-5}, \varepsilon=2^{-6}$.

parameter $\varepsilon$. Also, the method is first order convergent in the discrete maximum norm. Numerical example shows that recommended method has a good approximation characteristic as: in table and graphics, when $N$ takes increasing values, it is seen that the convergence rate of the smooth convergence speed $p^{N}$ is first order. The exact solution and approximate solution curves are almost identical as shown in Figure 1. In Figure 2, as $\varepsilon$ values decrease, the graph approaches more towards the coordinate axes in the boundary layer region around $x=0$ and $x=1$. In Figure 3, the errors in these regions are maximum because of the irregularity caused by the sudden and rapid change of solution in the boundary layer region around $x=0$ and $x=1$ for different values $\varepsilon$. Thus, numerical results prove that the proposed scheme is working very well.

\section{Competing interests}

The author declares that she has no competing interests.

\section{Authors contributions}

Author has contributed to all parts of the article. Author read and approved the final manuscript.

\section{References}

[1] Adzic, N., Spectral approximation and nonlocal boundary value problems, Novi Sad J. Math., 30, (3), (2000), 1-10.

[2] Amiraliyev, G. M., Difference method for a singularly perturbed initial value problem, Turkish J. Math., 22, (1998), $283-294$.

[3] Amiraliyev, G. M. and Cakir, M., A uniformly convergent difference scheme for singularly perturbed problem with convective term and zeroth order reduced equation, International Journal of Applied Mathematics, 2, (12), (2000), 1407-1419.

[4] Amiraliyev, G. M. and Cakir, M., Numerical solution of the singularly perturbed problem with nonlocal condition, Applied Mathematics and Mechanics (English Edition), 23, (7), (2002), 755-764.

[5] Arslan D., A new second-order difference approximation for nonlocal boundary value problem with boundary layers, Mathematical Modelling and Analysis, 25, (2), (2020), 257-270.

[6] Bakhvalov, N. S., On optimization of methods for solving boundary value problems in the presence of a boundary layer, Zhurnal Vychislitel'noi Matematikii Matematicheskoi Fiziki, 9, (4), (1969), 841-859.

[7] Bitsadze, A. V. and Samarskii, A. A., On some simpler generalization of linear elliptic boundary value problems, Doklady Akademii Nauk SSSR, 185, (1969), 739-40. 
[8] Cakir, M. and Amiraliyev, G. M., Numerical solution of the singularly perturbed three-point boundary value problem, International Journal of Computer Mathematics, 84, (10), (2007), 1465-1481.

[9] Cakir, M., Uniform second-order difference method for a singularly perturbed three-point boundary value problem, Advences in Difference Equations, (2010), 13 pages.

[10] Arslan, D., Stability and convergence analysis on Shishkin mesh for a nonlinear singularly perturbed problem with three-point boundary condition. Quaestiones Mathematicae, 43, (11), (2020), 1527-1540.

[11] Cakir, M. and Arslan, D., A numerical method for nonlinear singularly perturbed multi-point boundary value problem, Journal of Applied Mathematics and Phyics, 4, (2016), 1143-1156.

[12] Cakir, M. and Arslan, D., Numerical solution of the nonlocal singularly perturbed problem, International Journal of Modern Research in Engineering and Technology, 1, (5), (2016), 13-24.

[13] Cakir, M. and Arslan, D., Finite difference method for nonlocal singularly perturbed problem, International Journal of Modern Research in Engineering and Technology, 1, (5), (2016), 25-39.

[14] Chegis, R., The numerical solution of problems with small parameter at higher derivatives and nonlocal conditions, Lietuvas Matematica Rinkinys, (in Russian), 28, (1988), 144-152.

[15] Farell, P. A., Miller, J. J. H., O'Riordan, E. and Shishkin, G. I., A uniformly convergent finite difference scheme for a singularly perturbed semilinear equation, SIAM Journal on Numerical Analysis, 33, (1996), 1135-1149.

[16] Gupta, C. P. and Trofimchuk, S. I., A sharper condition for the solvability of a three-point second order boundary value problem, Journal of Mathematical Analysis and Applications, 205, (1997), 586-597.

[17] Herceg, D. and Surla, K., Solving a nonlocal singularly perturbed nonlocal problem by splines in tension, Univ. u Novom Sadu Zb. Rad. Prirod.-Mat. Fak. Ser. Math., 21 (2), (1991) 119-132.

[18] Miller, J. J. H., O’Riordan, E. and Shishkin, G. I., Fitted Numerical methods for singular perturbation problems, World Scientific, Singapore, 1996.

[19] Nayfeh, A. H. Introduction to perturbation techniques, Wiley, New York, 1993.

[20] O’Malley, R. E., Singular perturbation methods for ordinary differential equations, Springer Verlag, New York, 1991.

[21] Roos, H. G., Stynes, M. and Tobiska, L., Robust numerical methods singularly perturbed differential equations: ConvectionDiffusion and Flow Problems, Springer-Verlag, Berlin, 2008.

[22] Jankowski, T., Existence of solutions of differential equations with nonlinear multipoint boundary conditions, Comput. Math. Appl., 47, (2004), 1095-1103. 